\title{
Menschenbild und Menschenseele
}

\author{
Reto Eberhard Rast \\ Dr. med., Facharzt für Allgemeine Innere Medizin, Mitglied FMH, Präsident der Schweizerischen Gesellschaft für Nahtodstudien (SWISS IANDS)
}

Als mir einmal ein ostafrikanischer Arzt vorhielt, wir hätten in Europa kein Verantwortungsgefühl, war ich verdutzt. Wenige Tage zuvor war im Krankenhaus, in welchem wir beide tätig waren, ein junger Knabe verstorben, weil er in falscher Liegeposition war und ihm zu grosse Mengen Brei verabreicht worden sind. Dieser Tod hätte problemlos vermieden werden können, vor allem deshalb, weil bereits die vorletzte Verpflegung via Magensonde in die Lungen geflossen war und er nur mit Mühe vor dem Ersticken bewahrt werden konnte. Die Belehrungen seitens einer holländischen Ärztin hatten jedoch nichts gefruchtet: Am darauffolgenden Morgen wurde derselbe Fehler noch einmal begangen, und der Knabe verstarb. Dies ist nur ein Beispiel von mehreren Todesfällen, welche in diesem Krankenhaus mit etwas Aufmerksamkeit und einfachen Mitteln hätten vermieden werden können. Für die allermeisten dort tätigen Menschen liegt das menschliche Leben in Gottes-, nicht in Menschenhand. Man stirbt, wenn die Zeit dafür gekommen ist. Im erwähnten Fall des verstorbenen Knaben hatte die tansanische Pflegerin ihre Tätigkeit so ausgeführt, wie sie es gelernt hatte. Sie sah keine Notwendigkeit darin, dies zu ändern, das eigene Handeln kritisch zu hinterfragen.

\section{Vom Menschenbild des anderen lernen}

Mein kenianischer Kollege konnte diese Art von Verantwortung also nicht gemeint haben. Er sprach von der Verantwortung, die wir Europäer unseren alten Menschen gegenüber schuldeten. In Afrika würden die Kinder streiten, wer bei sich zu Hause die kranke Mutter pflegen dürfe. Unsere "Alterslager» waren für ihn der gleichwertige Verstoss wie für mich die hiesige Indifferenz gegenüber iatrogener Schädigung oder unprofessioneller Hilfeleistung. Beide Kulturen haben in ihrer Haltung gegen das Menschenbild der anderen verstossen. Beide tun gut daran, daraus zu lernen.

Die Stärke des «afrikanischen Menschenbildes» liegt nicht allein in der Grossmut gegenüber den Betagten. Hebt man den Stein des Anstosses, den wir Fatalismus nennen, kommt etwas Glänzendes zum Vorschein, das wir verloren haben, das wir aber zu unserem eigenen Heil in das moderne Menschenbild integrieren sollten. Den in vormodernen Kulturen aufgewachsenen Men- schen gilt das irdisch Sichtbare nur als Teil des Seins. Das Leben endet nicht mit dem Tod. Ein enormer Trost für den Vater, der still neben dem Knaben blieb, bis er gestorben war. Die hier als vormodern bezeichneten Kulturen haben nicht bloss Vertrauen in ein immaterielles Sein, sie sind selber vertraut mit jener jenseitigen Ebene, welche Teil ihres Alltags ist. Erscheinungen aus einer vermeintlichen Anderswelt gelten ihnen als normal, während wir sie als paranormal bezeichnen.

Aber nicht nur in Afrika, auch bei uns sind paranormale Phänomene in der Nähe des Todes so häufig, dass wir sie in diesem Kontext als normal bezeichnen müssen. Sie faszinieren mich, seit ich in meiner Primarschulzeit von meinem Schulpfarrer davon gehört habe. Ganz natürlich erzählte er von paranormalen Erfahrungen, die er als junger Soldat im Zweiten Weltkrieg gemacht hatte. Anders als die meisten seiner theologischen Kollegen war er darauf vorbereitet, als ihm bei einem Seelsorgebesuch im Spital Samedan ein schwerverletzter Patient berichtete, dass er, über der Unfallstelle schwebend, sich selber als ohnmächtigen Mann hätte liegen sehen. Vom Eintreffen des ihm bekannten Polizisten bis zum Krankentransport ins Spital habe er, schmerzfrei, alles von oben beobachten können. Solche später mit Nahtoderfahrung (NTE) bezeichneten Erlebnisse sind anderen Menschen kaum vermittelbar und zutiefst verunsichernd. Ablehnende Urteile oder Desinteresse gegenüber einer solchen Erfahrung ist die grösste Befürchtung eines Betroffenen im Kontakt mit anderen Menschen - und gleichzeitig die am häufigsten gemachte Erfahrung.

\section{Deutungshoheit paranormaler Phänomene}

Über die Deutungshoheit dieser paranormalen Phänomene wird gestritten. Für die monistisch oder naturalistisch geprägte Forschung entspringen solche Wahrnehmungen illusionärer Verkennung oder Halluzinationen und sind somit profaner Natur. Im Fall der oben beschriebenen ausserkörperlichen Erfahrung (AKE) wird eine Temporallappenreizung als Ursache postuliert, durch welche in seltenen Fällen ein als irreal empfundenes Gefühl, ober- oder unterhalb der aktuellen Liegeposition zu schweben, erzeugt werden kann [2, 3]. Für Idealisten oder Dualisten zeugen die vielen 
Anekdoten adäquater Wahrnehmungen während AKEs von einem transzendenten Bewusstsein, das unabhängig vom Körper existieren kann. Obwohl die Debatte von enormer Bedeutung für den Menschen ist, interessiert sich nur eine kleine Schar Wissenschaftlerinnen und Wissenschaftler dafür. Für die meisten Forscher ist die Fragestellung, ob AKEs real sind oder nicht, im besten Fall etwas beschämend, im schlechtesten ein Karrierekiller. Warum? Der aktuell dominierende Naturalismus schliesst eine transzendente Möglichkeit a priori aus. Da dieses Dogma aber implizit ist, wiegt sich die Mehrheit der Forscher in der Meinung, der Naturalismus sei keine Weltanschauung, sondern eine bewiesene Tatsache. Eine ernsthafte Auseinandersetzung kommt damit schnell ins Stocken, wenn sie nicht sogar mit Plattitüden abgewürgt wird. Von der AWARE-Studie [4], welche die Echtheit solcher AKEs mit Hilfe von Mustern, die an verschiedenen Orten von Intensivstationen so platziert wurden, dass sie nur von der Decke aus einsehbar waren, ein für alle Mal klären wollte, wird nicht selten behauptet, sie sei grandios gescheitert. Wie erwartet habe kein Betroffener während seiner NTE einen dieser Kästen wahrnehmen können. Aus Ignoranz oder sogar Kalkül wird verschwiegen, dass die einzige erfasste AKE gar nicht in der Nähe dieser Kästen stattfand, der dabei vom Betroffenen wahrgenommene Reanimationsablauf aber detailgetreu und korrekt wiedergegeben werden konnte.

Wenn reanimierte Patienten mit einer NTE im Vergleich zu solchen ohne NTE über ihre Reanimation detaillierte und korrekte Angaben [5], Blindgeborene eine Seherfahrung [6] und Patienten mit nachgewiesener ausgefallener Hirnfunktion akkurate Beobachtungen machen [7], wenn vollkommen andere Zeit- und Raumdimensionen erfahren werden können und das gesamte Leben auf einmal wahrgenommen sowie bewertet werden kann, wenn ausgerechnet bei Ausfall der Hirnrinde und im Unterschied zu Halluzinationen hochstrukturierte und hyperreale Erfahrungen mit Wirklichkeitsbezug gemacht werden können, wenn all diese Erfahrungen nicht in Worte zu fassen sind, weil ein irdisch vergleichbares Korrelat fehlt, wenn ein paar Sekunden irdischer Zeitrechnung die Betroffenen für das gesamte Leben prägen, wenn die Betroffenen auf dem immer wieder beschriebenen telepathischen Weg im Nachhinein verifizierte Informationen von Umstehenden oder von Verstorbenen aufnehmen können, wenn NTEs zu allen Epochen und in allen Kulturkreisen in denselben charakteristischen Erlebnismustern auftreten, dann sind solche Manifestationen sehr ernstzunehmende Hinweise für eine Transzendenz [8-10].

\section{Fällt das naturalistische Menschenbild?}

Piet van Spijk hat kürzlich in der Schweizerischen Ärztezeitung der Suche nach einem neuen Menschenbild die Tribüne bereitet [1]. Das freut mich. Die Zeit ist reif dafür. Er beklagt die Reduktion des Menschen auf ein berechenbares Maschinenmodell. Weiter findet er aber auch das dualistische Konzept von Descartes obsolet. Die Vorstellung einer Seele entbehre der Empirie. Zudem könne aufgrund ihrer Verschiedenheit der physische Leib gar nicht mit einer immateriellen Seele in Kontakt treten. Ein menschliches Unverständnis ist aber kein Beweis für deren Nichtexistenz. Bis heute wissen die Physiker auch nicht, was das Universum zusammenhält. Die dafür postulierte dunkle Materie ist genauso obskur, wie ihr Name es sagt. An den Grenzen der klassischen Physik werden die Phänomene paranormal. An der Grenze zum Tod geschieht dasselbe.

Ich gehe davon aus, dass das naturalistische Menschenbild früher oder später fallen wird, weil man die transzendenten Phänomene auf Dauer nicht wegdeuten und die Hypothese, dass Bewusstsein aus Materie emergiere, nicht bestätigen kann. Beim sukzessiven Übergang in ein neues Menschenbild kann der kartesianische Dualismus behelfsmässig in die Lücke springen, da er im westlichen Kulturkreis am besten bekannt ist. Nach Descartes sind Leib und Seele, materielles Gehirn und geistiges Bewusstsein zwei verschiedene Entitäten. Tiere und Pflanzen sind für ihn seelenlos, da sie wie die menschlichen Körper als eine von der Seele getrennte materielle Einheit rein mechanisch begründet werden. Diese Ansicht widerspricht modernen Kenntnissen [8], womit das kartesianische Menschenbild nicht als dasjenige der Zukunft angesehen werden kann. Auch sollte ein neues Menschenbild die von Piet van Spijk aufgezeigte Lücke des Dualismus, das fehlende Bindeglied zwischen Leib und Seele, schliessen können. Solche ontologisch-anthropologischen Modelle existieren. Hier sei abschliessend nur das vierstufige Modell des Quantenphysikers Burkhard Heim (1925-2001) genannt, das bisher noch wenig rezipiert wird. Heim postuliert ein Zusammenspiel von Physis (Körper), Bios (Lebenskraft), Psyche (Seele) und Pneuma (Geist) [11].

Ein transzendentes Menschenbild kann unserer Existenz die Sinnhaftigkeit verleihen, nach der wir suchen. Zurzeit spricht man nur noch in der Dritten Welt ungezwungen offen, heiter und nachdenklich zugleich über die aussersinnliche Welt. Die Forschung der sogenannt fortschrittlichen Welt muss die Frage danach stellen dürfen, ohne sich dafür schämen zu müssen. 